Revista de la red interuniversitaria de estudios sobre las literaturas rioplatenses contemporáneas en Francia argentina y chilena del siglo XX

\title{
Misticismo poético a ambos lados de la cordillera: Purgatorio de Raúl Zurita y Hospital Británico de Héctor Viel Temperley
}

Mysticisme poétique des deux côtés des Andes: Purgatorio de Raúl Zurita et Hospital Británico de Héctor Viel Temperley

Poetic mysticism on both sides of Los Andes: Purgatorio by Raul Zurita and Hospital Británico by Héctor Viel Temperley

\section{Adela Busquet}

\section{OpenEdition}

Journals

Edición electrónica

URL: https://journals.openedition.org/lirico/11372

DOI: $10.4000 /$ lirico. 11372

ISSN: 2262-8339

Editor

Réseau interuniversitaire d'étude des littératures contemporaines du Río de la Plata

\section{Referencia electrónica}

Adela Busquet, «Misticismo poético a ambos lados de la cordillera: Purgatorio de Raúl Zurita y Hospital Británico de Héctor Viel Temperley», Cuadernos LIRICO [En línea], 23 | 2021, Publicado el 19 diciembre 2021, consultado el 07 enero 2022. URL: http://journals.openedition.org/lirico/11372 ; DOI: https:// doi.org/10.4000/lirico.11372

Este documento fue generado automáticamente el 7 enero 2022.

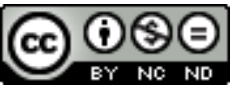

Cuadernos LIRICO está distribuido bajo una Licencia Creative Commons Atribución-NoComercialSinDerivar 4.0 Internacional. 


\title{
Misticismo poético a ambos lados de la cordillera: Purgatorio de Raúl Zurita y Hospital Británico de Héctor Viel Temperley
}

\author{
Mysticisme poétique des deux côtés des Andes: Purgatorio de Raúl Zurita et \\ Hospital Británico de Héctor Viel Temperley \\ Poetic mysticism on both sides of Los Andes: Purgatorio by Raul Zurita and \\ Hospital Británico by Héctor Viel Temperley
}

Adela Busquet

\section{Introducción}

Existe un consenso en torno a que la obra de Héctor Benjamín Viel Temperley (1933-1987) pertenece a las poéticas del misticismo. Tanto Hospital Británico (1986) como los ocho libros que lo preceden, lindan, según un punto de vista compartido por la crítica (Kamenszain, Monteleone, Muschietti, Mallol, Sylvester, Di Cío, Milone, Muzzio, Fara, Ioskyn), con la tradición de la poesía mística, a veces también denominada cultora de lo sagrado o, en algunos casos, religiosa (Molina, Fogwill, Piña). De igual modo, parece haber un consenso en torno al primer libro de Raúl Zurita (1950), Purgatorio (1979). Abonada, en parte por las declaraciones del propio poeta, se ha extendido la hipótesis de que los discursos de la lógica, la topología y la teoría de conjuntos constituyen los elementos vertebrales de esta obra. Esta vía de interpretación comienza con la reseña de Ignacio Valente, "Raúl Zurita: Purgatorio", publicada en el diario El Mercurio el 16 de diciembre de 1979, y se verifica en la conocida tesis de Rodrigo Cánovas, Lihn, Zurita, Ictus, Radrigán: Literatura chilena y experiencia autoritaria (1986), donde se afirma que en Purgatorio "no hay versos sino proposiciones lógicas" (59), y vuelve a aparecer en artículos más recientes como, por ejemplo, "Intertextualidad 
científica en Purgatorio de Raúl Zurita" (2013) de Alejandro Tarrab y "Raúl Zurita: entre la lógica y el desvarío" de Andrés Fischer (2014). El presente artículo no propone desdecir estos dos consensos. En efecto, los aparatos procedimental, léxico y sintácticoexperiencial en la obra de Viel Temperley dan crédito a la interpretación prevalente, mientras que los versos cuasisilogísticos de Purgatorio, muchos de ellos escandidos en forma de teoremas, vuelven innegable la apropiación zuritiana del campo de las matemáticas y la lógica formal. Lejos de objetar esas interpretaciones, intentaremos tensionarlas con el propósito de aventurar una continuidad entre lo místico y lo lógico.

2 Como se irá desarrollando en adelante, la diferencia entre los polos lógico y místico no se apoya en una distinción de tipo esencial (eidos, ousía) sino de grado. Es decir, si lo lógico, a primera vista, resulta antagónico de lo místico, es porque permanece oculta la gradación que conduce de una posición a otra. Más allá de los argumentos que en las sucesivas páginas daremos para integrar estos dos extremos, vale decir que al interior de Hospital Británico y Purgatorio existen vasos comunicantes que dan cuenta de tránsitos lógico-místicos comunes; esto es, interacciones no del orden intertextual, sino entre concepciones de vida y obra, entre proyectos poéticos que en última instancia responden a la misma tentativa de transitar lo intransitable.

3 Se parte, entonces, de la literalidad de los hechos: en la madrugada del 11 de septiembre de 1973, Raúl Zurita es detenido y torturado en Valparaíso, y luego trasladado al buque carguero Maipo donde pasa las siguientes treinta noches. Los manuscritos de sus primeros poemas, "Áreas verdes", son arrojados al mar por un soldado de la Armada. Con veintitrés años, dos hijos, sin trabajo ni estudios universitarios completos, y separándose de su entonces mujer, Miriam Victoria Martínez, se cierra simultáneamente una etapa en la jovencísima vida del autor y en la historia colectiva chilena. El 4 de mayo de 1974, ocho meses después de la irrupción de estos sucesos, Raúl Zurita es diagnosticado por la psicóloga Ana María Alessandri con "numerosos elementos positivos de psicosis de tipo epiléptico" (Zurita 2010: 47). La vida que emerge tras ese mes en el carguero Maipo es de una desesperación y crudeza extremas, en la que la experiencia del desfondamiento personal toca vértices psicológicos y humanos atravesados por la humillación y la pobreza. De este desasimiento dan cuenta los conocidos episodios de autoagresión que el poeta realiza en 1975 y en 1980: la quemadura de la mejilla y el intento de cegamiento, al arrojarse amoníaco sobre los ojos.

4 A la inversa de lo ocurrido en los jóvenes años del poeta chileno, la experiencia de desfondamiento en Viel Temperley se produce al final de su vida, a raíz -según cuenta el mismo autor- de la enfermedad y consecuente muerte de su madre, Cornelia Solari Parravicini, y en paralelo al tránsito por su propia enfermedad terminal: "Caí enfermo cuando vi que mi madre quería morirse" (1987: 59)². Hospital Británico es un libro escrito entre dos desvanecimientos: el desvarío producido por un tumor cerebral que será extirpado en el Hospital Británico de la Ciudad de Buenos Aires en 1985, y la muerte del autor en 1987. Según comenta su segunda mujer, Luisa Hansen, el segundo -y definitivo- episodio de pérdida de consciencia ocurre en el campo familiar de Parravicini mientras el poeta se encontraba hachando: "De un momento a otro se quedó seco, inmóvil (...) me hacía señas, no podía hablar" (Hansen en Bregazzi 2015: 123). Esa escena marca la aparición de un segundo tumor cerebral del que Viel Temperley ya no se recuperará. Es en esa ventana de tiempo, marcada por el desvarío y la muerte, que el autor escribe su último y terminal libro Hospital Británico ${ }^{3}$. 
5 A la luz de estos hechos biográficos, analizaremos el continuum lógico-místico como parte de una experiencia mayor conformada a la manera de un descenso. La continuidad entre un polo y otro, que podría figurarse por medio de una escala horizontal, incluye además un vértice inferior que integra el supuesto antagonismo en una verticalidad descendente, una especie de unidad catábica. Como si lo que hiciera posible que lo lógico y lo místico se encontraran íntimamente fuera un descenso, que, como veremos más adelante, será, desde otro punto de vista, un ascenso.

\section{El presente de la mística}

6 En el libro Mística y religión (2009), Vicente Fatone se detiene en la relación entre la mística y lo temporal: "La mística, más que cualquier otra actitud o sistema, afirma la dignidad del presente" (70). El aquí y ahora (hic et nunc) del presente místico "de todos los tiempos y culturas ha insistido en la necesidad de sacrificar la voluntad, que es en definitiva una manera de sacrificar el futuro" (2009: 69)4.

7 Como se ha señalado, tanto Purgatorio como Hospital Británcio son libros escritos en respuesta a acontecimientos radicales que irrumpen cancelando el tiempo por venir. En ese sentido, la experiencia del presente absoluto es anterior a ambos textos. Constituye su antecedente inmediato e inalienable; y sin embargo, también es interior a estas obras, como se comprueba en el plano enunciativo, donde impera el presente del indicativo a la par de una disposición fragmentaria y aforística mediada por discontinuidades temporales y lógicas (elipsis) que niegan toda linealidad narrativa, y en consecuencia, cronológica. La exposición a un presente abisal, por lo tanto, supone el primer anclaje o residencia en la mística de Purgatorio y Hospital Británico. Podría decirse que estos libros documentan el intento de residir aún en un tiempo suspendido; de afirmar, por medio de la escritura, la salida de una realidad infernal y el paso a un presente que participa nuevamente del lenguaje, es decir, de la vida ${ }^{5}$.

8 El presente terminal arranca a estos textos de la sucesión cronológica, y sin embargo, al estar anclados en experiencias concretas del dolor humano, no pueden escapar de la temporalidad, de las unidades irrevocables de principio y fin. La experiencia temporal que emerge es, entonces, propiamente purgatorial: un entremedio (aevum) entre dos temporalidades suspendidas: tempus y aeternitas (Kermode, 2000: 63). Al ser respuestas a un presente purgatorial, absoluto, la escritura de estos poemas se convierte en un proceso absolutorio que intenta purgar la vida-obra del rigor de las circunstancias, sea este el dolor producido por un diagnóstico médico terminal, o por la represión y la persecución militares.

9 La etimología de la palabra absoluto nos dirige en esta misma dirección. El verbo solvo explica Girogio Agamben- indica "la operación de soltar (luo) que conduce o reconduce algo al propio *se, es decir, al suus (...) soltándolo -absolviéndolo- de todo lazo y de toda alteridad" (2016: 149). Tanto Hospital Británico como Purgatorio son libros que se vinculan con lo absoluto en tanto implican la experiencia de "lo que a través de un proceso de 'absolución', ha sido reconducido a lo que es más propio, a sí mismo, a lo que es de él solo (...) a su soledad" (Agamben 2016: 149)6. Como dice Simone Weil: "Si nos contemplamos en un momento determinado -en el instante presente, seccionado del pasado y del futuro-, somos inocentes" (1994: 49). Y agrega: "Aislar de esa manera un instante supone el perdón. Pero ese aislamiento es desasimiento" (49). No hay duda de que el dolor es una nota esencial en ambos libros, en la medida en que la experiencia 
del desfondamiento o el tocar fondo subyacen a la escritura y a la estructura de estos poemas. Al mismo tiempo, este reconducirse a lo más propio, absolviéndose, liberándose de toda alteridad, es decir, de la implacable condena de las circunstancias, sean estas políticas o médicas, no puede ser visto sino como un gesto de redención ${ }^{7}$. Desde esta perspectiva, la escritura cobra la fuerza de un "ejercicio privado de resurrección", como señala el mismo Zurita (2016: 19). A propósito del vínculo entre dolor y escritura en Purgatorio, Ignacio Valente escribe:

El propio libro ofrece al lector algunas pistas documentales: el autocastigo fisico, el sufrimiento de una posible sicosis... Pero estos datos no son esenciales: la poesía misma tiene en el dolor su punto de partida. Y digo partida porque la experiencia integral de Purgatorio es un proceso espiritual que, naciendo del dolor, emprende una dificil ascensión que lo supera y lo redime, convirtiéndolo en algo parecido a la dicha y a la libertad (...). Es decir, el dolor inicial se transfigura en gloria precisamente a través de la escritura misma del poema: la redención se identifica con la poesía en acto (1980 Web).

Si el dolor implica un descenso, es decir, un desfondamiento, la resurrección que resulta del propio acto de escritura deja entrever que todo descenso es, en rigor, un ascenso hacia lo trascendente ${ }^{8}$. Es este mismo esquema de ascenso invertido el que estructura el viaje de Dante por los tres reinos ultraterrenos en la Comedia; bajar por los nueve círculos del infierno lo acerca, paradójicamente, al paraíso, a la visión de Dios. Este reconducirse -soltándose, absolviéndose de la condena de lo "excesivamente claro" (Viel en Bizzio 1987: 59), transformando las carencias en obras de arte (Zurita 2014: 72)'- puede ser pensado, en términos de Simone Weil, como el camino de la desgracia a la gracia: "Nuestro papel consiste en estar cara a cara a lo universal. (...) Es imposible mediante un movimiento ascendente, pero es posible mediante un movimiento descendente" (1994: 55).

11 Tres movimientos descendentes recorren la biografía de Viel Temperley, tres experiencias que lo arrancan de la sucesión temporal y lo exponen al absoluto del instante: cuando era niño y su madre lo lleva al río, cae al agua y casi muere ahogado: "Recuerdo que estaba sentado debajo del agua en paz, sin extrañar absolutamente la vida, (...). Y era feliz" (Viel Temperley en Bizzio 1987: 58); cuando habiendo comulgado "como un ahogado" (1987: 58) sale del Santísimo Sacramento hasta las barrancas de la plaza San Martín y el viento de Dios le pega en la cara: "Vengo de comulgar y estoy en éxtasis" (2013: 337); y finalmente, cuando en 1985, luego de ser operado del tumor cerebral, sale al patio del pabellón del Hospital Británico -que él llamó Rosetto- y la luz de Dios le "arruinó la vida en el mundo" (1987: 59). En esos descensos -que son también ascensos hacia lo absoluto- "lo que es completamente superior reproduce lo que es completamente inferior, pero invertido" (Weil 1994: 82).

12 El movimiento descendente en la obra y la vida de Raúl Zurita merece un trabajo aparte $^{10}$. Lo que en todo caso es importante remarcar es que el instante de inocencia -la absolución- en Purgatorio responde a un trasfondo histórico político en el cual la acusación, la humillación y la desaparición eran las fórmulas de la vida cotidiana, y es justamente a raíz de ese contexto que los versos: "Te amo -me dije- te amo // Te amo más que a nada en el mundo" (2010:17) se improntan con fuerza redentora. El absoluto de ese amor en ese presente total puede ser leído como el resultado del desasimiento que un hombre y una época alcanzan.

Resumiendo lo dicho hasta acá, podemos aventurar el siguiente esquema: la cancelación del futuro engendra la exposición a un presente absoluto. La principal 
inscripción mística en Purgatorio y Hospital Británico es precisamente la dignificación del presente. Segmentado el presente del futuro y el pasado, se instaura un tiempo purgatorial donde el camino de la absolución, es decir, de la liberación respecto de las circunstancias en el umbral entre la vida y la muerte, se produce por medio de la escritura. Este proceso de liberación tiene la estructura de un descenso hacia lo alto, hacia lo trascendente.

\section{El continuum lógico-místico}

A diferencia de la religión, que supondría "el sentimiento de absoluta dependendencia" (Fatone 2009: 36), la experiencia mística "puede ser definida como un sentimiento de independencia absoluta" (36). Partiendo de esa suerte de autonomía, Fatone intenta demostrar que "la mística supone una liberación del pensamiento", a la vez que su lógica "se articula con otras lógicas" (37). Con ese propósito define cuatro momentos por los cuales transcurre el pensamiento, siendo el último de todos el estadío místico. $\mathrm{Si}$, como dijimos antes, los polos místico y lógico participan de una continuidad no siempre evidente, la pretensión de este apartado es explicitar de qué modo los distintos momentos del pensamiento (prelógico, lógico formal, lógico dialéctico y místico) conviven en Purgatorio y Hospital Británico, expresando finalmente esa continuidad que, en última instancia, es la respuesta a un descenso convertido -o absuelto- por medio de la escritura, en un ascenso.

El primer estadio del pensamiento se denomina prelógico y corresponde al sueño que "debe resolverse en la simplicidad de la afirmación ingenua. Todo en el sueño es y es presente" (Fatone 2009: 37). En la dedicatoria de Hospital Británico se lee un verso que luego se repite en el primer poema: "Mi madre es la risa, la libertad, el verano" (2013: 370 y 371). De manera análoga, en un poema de Purgatorio, titulado "Como un sueño", Zurita escribe los siguientes versos: "YO USTED Y LA NUNCA SOY LA VERDE PAMPA / EL DESIERTO DE CHILE" (2010: 27). Este lenguaje, que establece proposiciones en forma de juicio por medio de cópulas, forcluye tanto el movimiento traslaticio de la metáfora como la mediación del símil, cuya apoyatura en el adverbio "como" revela ya el pliegue de una negación. Todo en este primer momento es. No hay reverso todavía, no existe la negación porque, como dice Weil: "Para salir del sueño hay que tocar la imposibilidad. En sueños, no existe la imposibilidad. Solamente la impotencia" (1994: 79).

El segundo momento, señala Fatone, se denomina formal, y en él se descubre la negación (2009: 37). La oposición dualista de la lógica formal emerge en la medida en que se desvanece la unidad prelógica primordial y la realidad se estructura en términos de contrarios.

i. Los desiertos de Atacama son azules

ii. Los desiertos de Atacama no son azules ya ya dime lo que quieras

iii. Los desiertos de Atacama no son azules porque por allá no voló el espíritu de J.

Cristo que era un perdido (...) (2010: 36).

Los versos pertenecen al poema "El desierto de Atacama III" de Purgatorio y pueden leerse como un guiño que refracta la persecución mental engendrada por el discurso regido por el principio de no contradicción. Este lenguaje de blanco o negro demanda que la enunciación se resuelva por un término o por el otro de modo disyuntivo, generando claridad a expensas del violento contraste con el término excluido. Si bien la incorporación de este lenguaje lógico-formal tiene efectos poéticos y constituye una de 
las innovaciones del poemario ${ }^{11}$-en el sentido de que permite ir más allá de las poéticas parriana y nerudiana-, su presencia se lee también por fuera de la página, es decir, en continuidad con la época. Esa es la función de estas incrustaciones esquizo-dualistas: el término excluido es el mismo pueblo excluido y la exigencia de definiciones claras y distintas presupone ya el triunfo de un lenguaje reducido a su expresión interrogatoria. El yo poético de Purgatorio es, por un lado, un perseguido, y eso se evidencia en la paranoia discursiva expresada en términos lógico-formales, pero también en la incorporación del ya referido diagnóstico de la psicóloga Alessandri. Lo que el poeta evidencia al incluir e intervenir este documento es la violencia de la interpretación en juego: la dualidad entre locura y normalidad, entre incluido y excluido, entre el paciente del discurso científico y el hombre de la experiencia histórica -dictadura mediante- en el análisis del sufrimiento humano. Contra esa interpretación dualista, que podríamos encasillar en el segundo momento del discurrir lógico, es decir, en la fase formal, se yergue Purgatorio y en particular las tachaduras que el poeta realiza en el documento psiquiátrico. De igual modo pueden leerse los encefalogramas incluidos hacia el final del libro. Sin embargo, este poemario inicial anuncia un elemento redentorio que insistirá a lo largo de toda la obra zuritiana: en medio de esa rotura psíquica y social, el yo poético afirma un amor exponencial cuya fuerza congregante vuelve a unir los fragmentos de ese "yo roto o disperso" por la violencia de la exclusión.

En Hospital Británico, en cambio, este lenguaje de verdades sostenidas en la anulación de lo contrario, no responde al discurso del interrogatorio militar, sino a las definiciones "excesivamente claras" del diagnóstico médico. Hospital Británico es una tentativa de evasión, de absolución, de liberación con respecto a la clausura que introduce ese lenguaje dualista en la intimidad de una vida. Por eso, el discurso lógico-formal no se lee dentro de la obra, sino en la única entrevista otorgada por el autor, que funciona a la manera de un paratexto aclaratorio:

V. T. -(...) empezó a interesarme la poesía que me permitía no solamente esconderme sino evadirme y hacer un mundo, tener un mundo.

S. B. - ¿Evadirte de qué?

V. T. -De lo excesivamente claro. (...) "Hospital Británico" me permite creer que me salí del mundo y no sé para qué. (1987: 59)

Hospital Británico advierte el límite del pensamiento dualista y lo supera. La certeza formal propia del diagnóstico médico es indiferente al contexto de recepción, porque su excesiva voluntad de determinación barre la posibilidad de otra interpretación y aun de la fe, que como dice Hebreos 11: "Es pues la sustancia de las cosas que se esperan, la demostración de las cosas que no se ven". Disputarle a los hechos su condición de únicos es finalmente lo que se lee en este libro; el enfermo oncológico Viel Temperley se libera del rótulo "enfermo terminal" dignificando, como sucede en la mística, su presente por medio de un lenguaje que incluye la indeterminación, la esperanza en lo desconocido: "Fui rodeado y traspasado por una sensación de amor tan intensa que me arruinó la vida en el mundo" (Viel Temperley en Bizzio 1987: 59).

El lenguaje dualista, sostenido en el esquema de los contrarios excluyentes, sin embargo da pie a un plano que excede justamente la dualidad. Ese plano es lo imposible, lo trascendente. No nos referimos con este término solo a la comunión con Dios (Viel Temperley), sino además, a lo contrario; esto es, la experiencia de su ausencia (Zurita). Aun ausente, Dios o lo trascendente o lo imposible, abre un más allá en la creación poética. 
21 Simone Weil se detiene, precisamente, a pensar esta cuestión en los apartados "Lo imposible" y "Contradicción" de La gravedad y la gracia (1947). En primer término, distingue "los contrarios" de los "contradictorios". Weil escribe: "La correlación representable de los contrarios [alto y bajo, leve y pesado, dulce y amargo] es una imagen de la correlación trascendente de los contradictorios" (1994: 81). Podemos leerlo así: los contrarios tensan los polos opuestos de la racionalidad refractando así la contradicción que despierta o descubre el plano trascendente. La experiencia de los contradictorios -es decir, la comunión de términos opuestos- nos revela lo trascendente, lo imposible. Continuando este camino, agrega: "Lo que puede la relación entre contrarios en vistas a tocar al ser natural, lo pueden los contradictorios considerados en su conjunto con vistas a tocar a Dios" (81). Y luego: "La contradicción sentida en el fondo del ser es desgarro, es la cruz" (81). Es por eso que la experiencia de la contradicción conlleva una especie de abajamiento hacia lo alto ${ }^{12}$. Los versos que inauguran Purgatorio y su dedicatoria hacen chocar los contrarios alumbrando el plano de la contradicción y a través de ella, lo trascendente: "Mis amigos creen que / estoy muy mala / porque quemé mi mejilla"; "DEVOCIÓN // A Diamela Eltit: la / santísima trinidad y la / pornografía // "LA VIDA ES MUY HERMOSA, INCLUSO AHORA"” (2010: 7 y 9). El choque entre el yo poético y el yo del autor queda expresado en la flexión femenina del adjetivo "mala" que, ni bien abierto el libro, hace aflorar una primera contradicción. Así también ocurre con los polos teológico y profano (dogma de fe y dogma de la carne) que la dedicatoria tensiona, donde el verso final en mayúsculas explicita la contradicción entre la belleza de la vida y el horror de la época. Esto último, vuelve a mostrar que la contradicción -lo imposible-se vincula tanto con lo trascendente como con la experiencia del extremo dolor: "La vida humana es imposible. Pero solo la desgracia logra que lo sintamos" (Weil 1994: 79). El dolor hace patente lo imposible. Tal como suele decirse: Dios -o su ausencia- se presenta sobre todo ante quienes sufren. Por las experiencias biográficas que atraviesan ambos autores, ese desgarro es la coordenada cero de Purgatorio y Hospital Británico.

Es así como llegamos al tercer momento del pensamiento. Este estadio se denomina lógico porque corresponde a la dialéctica, que, como se sabe, se trata de afirmar y negar a la vez. De este modo pueden leerse los siguientes versos de Hospital Británico y Purgatorio respectivamente: "Cristo es Cristo madre, y en Él viene mi madre a visitarme" (2013: 383); "Por eso lo que está allá nunca estuvo allá y si ese / siguiese donde está vería darse vuelta su propia vida (...)" (2010: 39). El propósito de estos enunciados ya no consiste en presentar dos premisas contrarias y excluyentes, sino en albergar en la unidad de la contradicción lo aparentemente irreconciliable. Es en este momento dialéctico donde, al tensionar las posibilidades del lenguaje ${ }^{13}$, se inaugura un plano trascendente tal como lo expresa Simone Weil, y se les arrebata a los hechos del mundo su condición de incontestables.

El avance por medio del lenguaje hasta las fronteras mismas de la experiencia nos enfrenta a la noción de límite, específicamente el límite entre lo que llamamos lenguaje y aquello que deja de serlo ${ }^{14}$. El poeta y lingüista peruano Mario Montalbetti dedica varias páginas de su libro El pensamiento del poema (2019) a este concepto. En el apartado número nueve esquematiza "una figura (imaginaria, imposible) del lenguaje como objeto cerrado (clausurado por la semántica)" con el fin de pensar cuáles son sus fronteras (2020: 92). El poema y el matema son aquellos opuestos que al ubicarse en la periferia del lenguaje estarían en el lugar de la intersección entre el lenguaje y aquello 
que ya no lo es (92). Mientras que la prosa y la ciencia se encuentran dentro del conjunto imaginario "lenguaje", el poema y el matema delimitan el conjunto y constituyen sus respectivos bordes. En continuidad con las ideas de Montalbetti, podemos añadir que la mística también se encuentra en el umbral de esa bolsa imaginaria que llamamos lenguaje. Digámoslo así: poema, matema y mística hacen borde entre lenguaje y no lenguaje.

Algo semejante afirma Fatone al desarrollar el cuarto momento, que, como puede anticiparse, se trata de la negación del estadio dialéctico, tal como se aprecia en el famoso fragmento de Heráclito: "El Uno (...) quiere y no quiere ser llamado por su nombre" (2009: 39). “Así se instauró (...) la lógica apofática propia de la mística: negando aquel no quiere y quiere para convertirlo en esto otro: ni quiere ni no quiere" (39) ${ }^{15}$. La mística supondría un paso ulterior respecto a la dialéctica hegeliana. "No es así, no es así (neti, neti)", es la respuesta mística ante cualquier afirmación o negación (39).

Los poemas "Las llanuras del dolor" y "Mi amor de Dios" de Purgatorio, pueden leerse a la manera de una instancia mística donde lo verbal cede lugar a lo visual. En el primero, el nombre de Dios (Eli, "padre"), escrito sobre los ejes cartesianos, desciende hasta borrarse donde aparece el verso final "y dolor" fuera de la coordenada horizontal. A propósito de este dibujo, William Rowe comenta: “Cómo leer esto? ¿No sería que el dolor aparece precisamente como un exceso en relación con la lógica del espacio cartesiano, ocupada por las palabras sagradas?" (2017: 15); en la segunda imagen, los peces reunidos en forma de pirámide invertida refractan, quizás, el milagro de la multiplicación de los panes y los peces en el mar de Galilea ${ }^{16}$. Ambos poemas sugieren un "es así, es así", que termina negándose en la sugerida ausencia de Dios y de la distribución del alimento, a la manera de un: "No es así, no es así"; es decir, no sucede así en el mundo.

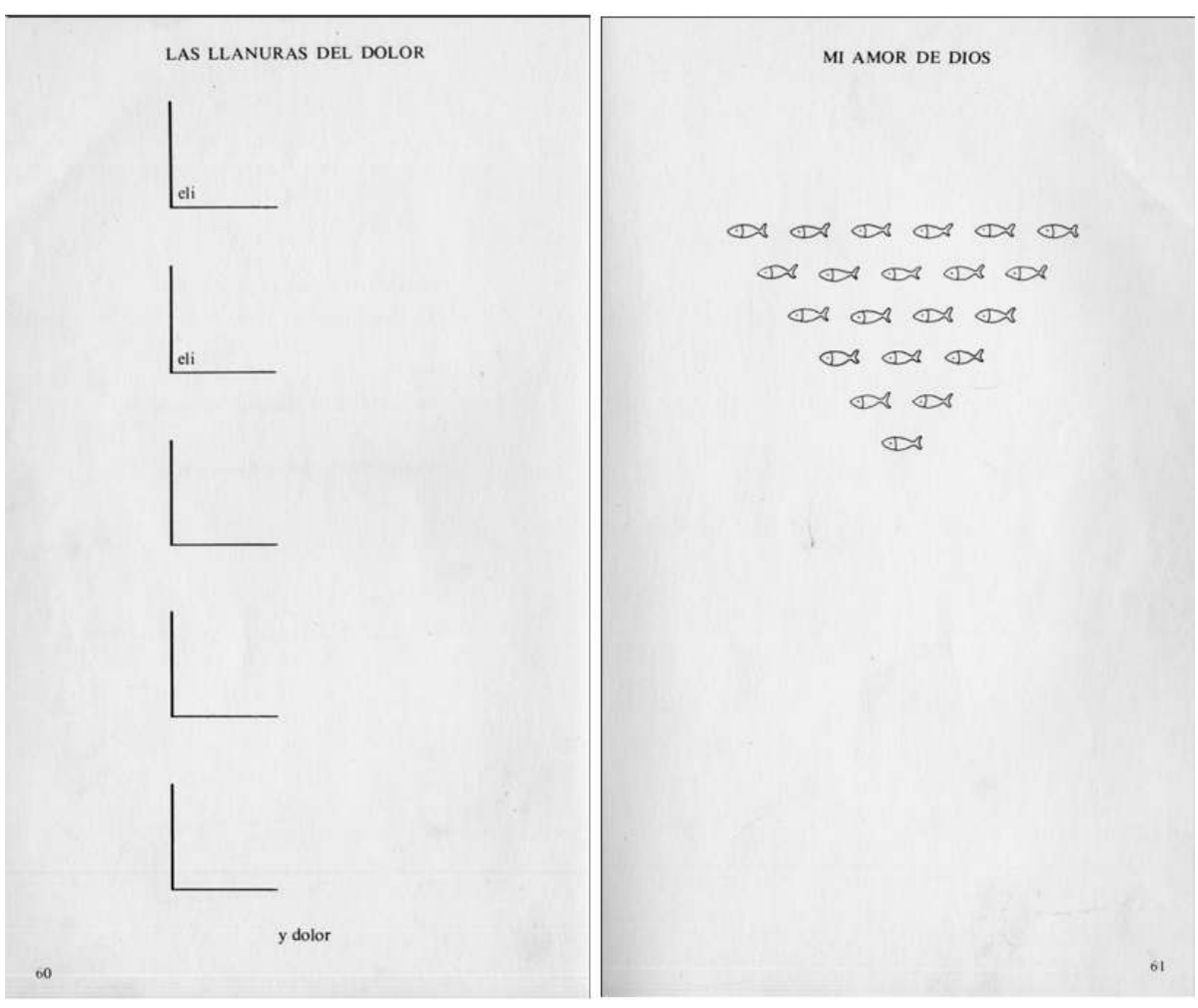


26 En esta misma dirección puede leerse el último verso de Hospital Británico, que el autor recupera del poema "Qué calor hace, madre" publicado en Humanae vitae mia (1969). A la luz de la muerte de su madre, los versos "El verano en que resucitemos / tendrá un molino cerca / con un chorro blanquísimo / sepultado en la vena" (2013: 94), vuelven a aparecer en Hospital Británico ya sin la escansión heptasilábica original, dispuestos en un solo período sintáctico sin cortes y con la inclusión de la fecha de escritura original entre paréntesis: (1969). Sobre esto mismo, Mattoni apunta algo más: "La misma traslación a prosa (...), señala este alejamiento de la poesía de toda la vida que para Viel significaba, acaso sin una voluntad definitiva, el alejamiento literal de la vida" (2011: 89). Pero incluso el contenido semántico del poema nombra un alejamiento definitivo: la esperanza de resurrección en la evidencia de la muerte. A lo coloquial de la expresión "el verano", como si fuera un verano más, se adjunta "en que resucitemos". Es decir, la excepcionalidad única o última o la insondable vez. Esa vez tendrá un molino cerca: algo común, el agua de todos los días. Pero será "un chorro blanquísimo". De nuevo, algo excepcional en el más común de los paisajes: esa agua de ese molino cerca ese verano, estará sepultada en la vena. El verso resucita y sepulta, habla de lo extraordinario y de lo más ordinario del mundo, del agua de todos los días como si fuera Dios mismo, blanquísimo, sepultado como la sangre nuestra (y no suya) para siempre en la vena, alguna vez bajo la tierra. Este movimiento pendular que va de lo cotidiano a lo excepcional en un solo verso, puede leerse en la línea del ímpetu místico de este último momento donde los opuestos afirmados se niegan a la vez. No se trata de un silencio, sino del "ni quiere ni no quiere" 17 apostado en el ir y venir de los contrarios reunidos en un vértice escatológico donde: "Los cabos que no podemos atar son testimonio de lo trascendente" (Weil 1994: 79).

Cristo, ni muere ni no muere; resucita de entre los muertos, pasa una breve temporada entre los vivos y asciende finalmente a los cielos. Lázaro, ya muerto, vuelve a la vida todo vendado ${ }^{18}$ y al poco tiempo vuelve a morir. Así Viel Temperley, luego de la operación del año 85, es atravesado por Dios en el patio del Hospital Británico y con la cabeza vendada vuelve a la vida para escribir -sin entender bien cómo- su último libro. Esta obra, como se dijo, está escrita entre dos desasimientos: ni vivo del todo, ni del todo muerto, sino justo en el medio purgatorial entre ambos estados, como un lapso de tiempo en el que el autovaciamiento (kénosis ${ }^{19}$ ) o distancia desamarrada del mundo anticipa la plenitud (pleroma) alcanzada en esa cruz; una suerte de vilo y resurrección ${ }^{20}$.

\section{Obras terminales: la escatología en el joven Zurita y el último Viel}

Si, como se dice en el evangelio de Mateo (20:16), "los últimos serán los primeros”, a la inversa, podría decirse también que lo primero será lo último ${ }^{21}$. Purgatorio es un libro inicial en un doble sentido: es el primero, escrito entre los veintitrés y veintinueve años del poeta, pero también es un libro que escribe una génesis personal. Esa génesis contiene la fuerza del fiat lux que crea algo donde había pura nada o ruina o escombro psíquico e histórico a la vez. Una revelación, es decir, un apokálypsis: "Me amanezco / Se ha roto una columna" (2010: 15), verso que nombra el final del lenguaje monumental de la poética nerudiana y su hercúleo esfuerzo por vertebrar poéticamente América en su triple dimensión histórica, lingüística y territorial ${ }^{22}$. A estos dos versos les sigue: "Soy una santa digo" (15), como diciendo: "Soy otro", o más bien, "otra". La fuerza de ese 
decir rimbaudiano inicial (Rowe 2017:16) está preñado de lo último: el quiebre o el fin del lenguaje épico nerudiano pero también, en la vereda opuesta, el final de la antipoesía de Parra ${ }^{23}$. Purgatorio expone la necesidad de otra poética que sea capaz de expresar la perforada hondura de los recientes hechos sudamericanos y en ese punto, condensa el final de una época y el comienzo de un nuevo lenguaje ${ }^{24}$.

En cuanto a su inscripción en el misticismo, hay una diferencia entre el lenguaje místico de este libro y las así llamadas "místicas tradicionales" (Milone 2014: 169). La particularidad del misticismo zuritiano radica en la imposibilidad de ser catalogado bajo la fórmula de la "experiencia interior". El éxtasis de Purgatorio no refiere a un "salirse de sí mismo" como la etimología de la palabra indica. Por el contrario, se trata de un intento de volver a armar la identidad perdida por medio de la subjetivación de un nuevo lenguaje (Rowe 2017: 19). El sí mismo de la interioridad de la conciencia llámese la psiquis o el alma- ha estallado a la par de los hechos. Como se dijo, el Golpe de 1973 no solo quiebra la continuidad del proceso histórico colectivo, sino también la integridad psíquica del poeta. A sus setenta y un años, Zurita vuelve a confirmar que el episodio de autoagresión del año 1975 -episodio que está en el comienzo de Purgatorio-, dio inicio a su obra poética, ya que "el dolor es una forma de volver a agruparse, de reunirse consigo mismo" (Zurita $2021 \mathrm{Web)}$ ). Este libro evidencia el esfuerzo del poeta por congregarse nuevamente y resistir la locura de la disgregación. Careciendo entonces de una interioridad como punto de partida, la mística de Purgatorio se despliega en el ámbito de la exterioridad; como si el lenguaje de las matemáticas, la lógica y el paisaje de Chile hiciera posible una reconstrucción de la identidad individual y colectiva ${ }^{25}$.

Hospital Británico, en cambio, representa la culminación de una poética que, según Eduardo Milán, "trae al escenario poético un faltante durante mucho tiempo en lengua castellana: la poesía mística" (2013: 40) ${ }^{26}$. El autor explica que tuvo la intención de romper su poesía porque en sus anteriores libros "la notaba demasiado rígida, como atada a un molde, un principio, un medio, un fin: sabía qué iba a decir" (1987: 59). En este sentido, la "lírica terminal" (2013: 7) de esta obra -tal como la denomina Tamara Kamenszain- representa un giro respecto a los ocho libros anteriores del poeta. Desde Poemas con caballos (1956) hasta Crawl (1982), Viel da cuenta de un sí mismo consolidado; un "yo" homologable a esa "x vacía" que -según Kant- debe poder acompañar todas sus representaciones, devolviéndole la conciencia de que efectivamente le pertenecen. Si bien es cierto que su poética se caracteriza por el protagonismo de los elementos exteriores -el mar, el revólver, el hacha, la hoja de afeitar-, todos ellos forman parte del éxtasis de un "yo poético" insuflado de trascendencia y misterio que recuerda a la denominada "experiencia interior" de la mística tradicional. Pero a diferencia de esa experiencia interior, Hospital Británico inaugura un quiebre que lo acerca a Purgatorio: al ser el testimonio de un trepanado, la interioridad del yo, hasta entonces garantizada físicamente por la circunferencia del cráneo, se dispersa e inicia una obra "en esquirlas" (Monteleone 2011: 102). La construcción formal del libro, basada en el recorte y la incrustación de versos ya publicados, refracta el procedimiento de corte del instrumento que abrió el cráneo del poeta: "Santa Reina de los misterios del hacha y de las brazadas lejos del espigón: Ruega por mí que estoy en una zona donde nunca había anclado con maniobras de Cristo en mi cabeza. (1985)" (Viel Temperley 2013: 382). Los versos obsesivamente fechados, parecieran indicar tanto "fechas posibles para resucitar" (Kamenszain 2013: 8) como la necesidad de reunir las astillas de una vida por 
primera vez rota: “¿Quién carajo armó todo eso? No tengo idea" afirma el autor meses antes de morir (1987: 59).

\section{Palabras finales}

En la interrupción de la vida, en momentos necesariamente escatológicos, el lenguaje parecería tomar conciencia de su sentido último y primero, su último y primer decir, donde lo absoluto queda en primer plano: de ahí la mística. El autoritarismo de los discursos -represión y psicosis en uno, cáncer en el otro- pone en evidencia una "herida abierta sin sutura" que vuelve necesario el plano trascendente (Rowe 2017: 15). Con casi diez años de diferencia, cada libro encierra una tentativa de reintegrar el misterio al lenguaje y la dignidad al presente, interrumpiendo el determinismo médicopolicial del diagnóstico para que de este modo, la poética redentora lleve hasta sus últimas consecuencias el hálito mistérico que permita "evadirse de lo excesivamente claro" (1987: 59).

Si bien Zurita hace uso explícito de la lógica formal y Viel Temperley es considerado parte de los poetas argentinos que integran la poesía mística (Cárcano 2020: 17) ${ }^{27}$ cuya obra es "una de las pocas obras místicas escritas en su siglo" (Sylvester 2011: 138) ${ }^{28}$, la voz que cuece a ambos libros es declaradamente escatológica, final, crepuscular y en el mismo sentido, fundante, instauradora de un lenguaje nuevo. Por eso, estos libros catábicos reúnen, justamente, la fuerza del comienzo: transitan por el continuum lógicomístico y, estando en la cruz, en la intensa plenitud de lo abandonado, se arrojan a la experiencia de "comenzar todo de nuevo". Así, ambas obras dan cuenta de esa independencia absoluta, ese desasimiento o desamarre absoluto que, como dijera Novalis, es la experiencia de "estar solos -y añadimos, desechas- con todo lo que amamos"29.

\section{BIBLIOGRAFÍA}

Agamben, Giorgio, El lenguaje y la muerte. Un seminario sobre el lugar de la negatividad, España, Gredos, 2016. Traducción de Tomás Segovia.

---, Altísima pobreza. Reglas monásticas y formas de vida, Buenos Aires Adriana Hidalgo, 2018.

Traducción de Flavia Costa y María Teresa D’Meza.

Anselmi, Max, "La memoria de la pasión mesiánica en la perspectiva de la espiritualidad. La investigación de Stanislas Breton”, «Stauros» Teología de la cruz, no 41, 2004, Web. Consultado 22 de marzo de 2021.

Arancet Ruda, María Amelia. "Héctor Viel Temperley: otro stalker en la estela del carmelita y su crawl sin descanso." Actas de las Jornadas de Literatura, Crítica y Medios: perspectivas, Buenos Aires, Universidad Católica Argentina, 2003, Web. Consultado 22-02-2021.

Auerbach, Erich, Figura, Madrid, Trotta, 1998 [1938]. Traducción de Yolanda García Hernández y Julio A. Prados. 
Badiou, Alain, El siglo, Buenos Aires, Manantial, 2005 [2005]. Traducción de Horacio Pons.

Bizzio, Sergio, “Viel Temperley: estado de comunión”, Vuelta Suramericana nํㅗ 12, Buenos Aires, 1987, 58-59.

Bregazzi, Juan Martín, “Héctor Viel Temperley: el poeta de la periferia. Reconstrucción biográfica, mitología y trayectoria en el campo literario del autor argentino", Tesis de Licenciatura, Buenos Aires, Universidad de Buenos Aires, 2015.

Brito, Eugenia, Campos minados. Literatura post-golpe en Chile, Cuarto propio, Santiago, 1990.

Busquet, Adela, (2017) "No es un hombre, es un tipo roto en un país roto. La poesía por fuera del cálculo yoico", "Quién" o "Qué". Los tránsitos del pensar actual hacia la comunidad de los vivientes, Mónica B. Cragnolini (comp.), La cebra, Buenos Aires, 2017, p. 179-199.

---, (2019a) “El poema, algunas notas entre Maurice Blanchot y Raúl Zurita”, Maurice Balnchot, fragmentos para una filosofía, Noelia Billi (comp.), Prometeo, Buenos Aires, 2019a, p. 97-108.

---, "Vínculos entre la poética de Raúl Zurita y algunas ideas de Jacques Derrida", Instantes y azares no 23, La cebra, Buenos Aires, 2019b, p. 197-215.

---, “Zurita o el poema exponencial de la piedad", ZuritaX70, Paulina Wendt (comp.), Editorial Mago editores, Santiago, 2021. (En prensa).

Busquet, Adela y Brockenshire, Lucas, “Zurita: una poética suramericana del paraíso”, Revista Chuy, 2021. (En prensa).

Cánovas, Rodrigo, Lihn, Zurita, Ictus, Radrigán. Literatura chilena y experiencia autoritaria, Flacso, Santiago, 1986.

Cárcano, Enzo, "Con los ojos en la noche”; La poesía mística de Jacobo Fijman en los márgenes, Universidad del Salvador, Buenos Aires, 2020.

De Rougemont, Denis, El amor y Occidente, Kairós, Barcelona, 1984. Traducción de Antoni Vicens.

Di Cío, Mariana "Viel temperley, hombre al agua", Poéticas del presente. Perspectivas críticas sobre la poesía hispánica contemporánea, Omar Ette y Julio Prieto (eds.), Iberoamericana Vervuert, España, 2016, p. 223-236.

---, "Zurita: el poema como memorial del dolor", América. Cahiers du Criccal. Poétiques et politiques de la mémoire en Amérique latine, 2018, p. 62-73.

---, "Las llagas abiertas de Raúl Zurita”, Fronteras, límites, intercambios en la obra de Raúl Zurita: un viaje por los meandros de la creación poética, Benoît Santini (dir.), Presses Universitaires du Midi, Toulouse, 2019, p. 51-62.

Fatone, Vicente, Mística y religión, Las cuarenta, Buenos Aires, 2009.

Fara, Daniel, "El bosque de arena”, Viel Temperley, Del Dock, Buenos Aires, 2011, p. 36-53.

Fischer, Andrés, “Raúl Zurita: entre la lógica y el desvarío”, Biblioteca Virtual Miguel de Cervantes, Alicante, 2014.

Fogwill, Rodolfo E., “Héctor Viel Temperley”, Diario de Poesía no 19, 1991, p. 35-27.

Jorge, Gerardo, "Dilemas en la poesía argentina de los años cincuenta", Exlibris n 1 1, Universidad de Buenos Aires, Buenos Aires, 2012, p. 283-298.

Kamenszain, Tamara, Prólogo, Héctor Viel Temperley. Obra completa, Del Dock, Buenos Aires, 2013, p. 7-10. 
Kermode, Frank. El sentido de un final, Gedisa, Barcelona, 1983. Traducción por Lucrecia Moreno de Sáenz.

Mallol, Anahí, "Mi Viel: de ángeles y aleluyas hasta el silencio, hasta la nada”, Viel Temperley, Del Dock, Buenos Aires, 2011, p. 64-80.

Mattoni, Silvio, "Nueve libros en busca de un estilo", Viel Temperley, Del Dock, Buenos Aires, 2011, p. 88-98.

Milán, Eduardo, Visiones de cuatro poemas y el poema que no está, Mangos de Hacha, México, 2013.

Milone, Gabriela, Héctor Viel Temperley. El cuerpo en la experiencia de Dios, Ferreyra Editor, Córdoba, 2003.

---, Pensamiento filosófico y experiencias religiosas en la poesía argentina contemporánea, Tesis de Doctorado, Universidad Nacional de Córdoba, 2004.

Montalbetti, Mario, El pensamiento del poema, N direcciones, Buenos Aires, 2020.

Monteleone, Jorge, “El cuerpo como profecía”, Viel Temperley, Del Dock, Buenos Aires, 2011, p. 99-109.

Muschietti, Delfina, "Viel Temperley: un texto mareador en el que todo renace", Viel Temperley, Del Dock, Buenos Aires, 2011, p. 110-120.

Muzzio, Diego, "Héctor Viel Temperley, el nadador vertical", Hablar de poesía $\mathrm{n}^{\circ} 4$, Grupo editorial Latinoamericano, Buenos Aires, 2000, p. 18-33.

Piña, Cristina, "Viel Temperley, el místico extraterritorial”, Viel Temperley, Del Dock, Buenos Aires, 2011, p. 121-135.

---, “Héctor Viel Temperley: de márgenes, exclusiones y extraterritorialidades”, Gramma oㅡ 50, 2013, p.136-150.

Sylvester, Santiago, “¿Un místico entre nosotros?”, Viel Temperley, Del Dock, Buenos Aires, 2011, p. 136-142.

Tarrab Rivera, Alejandro, "Intertextualidad científica en «Purgatorio» de Raúl Zurita", Biblioteca Virtual Miguel de Cervantes, Alicante, 2013. Consultado 22-02-2021.

---, “«Purgatorio», el lugar natural de las artes”, Biblioteca virtual Miguel de Cervantes, Alicante, 2014. Consultado 23-02-21.

Rowe, William. "Tiempo y medida en Purgatorio de Raúl Zurita”, Mitologías hoy, Vol. 15, 2017, pp. 13-20, Consultado 22-03-2021.

Valente, Ignacio, “Algo más sobre Zurita”, El Mercurio, 19 de octubre 1980, p. E3. Consultado 9-03-21.

Weil, Simone, La gravedad y la gracia, Trotta, Madrid, 1994. Traducción de Carlos Ortega Bayón. Viel Temperley, Héctor, Obra completa, Buenos Aires, Del Dock, 2013.

Zurita, Raúl, Literatura, lenguaje y sociedad: 1973-1983, Santiago de Chile: Comunicación y Cultura para el desarrollo, 1988 [1983].

---, Anteparaíso, Editores Asociados: Chile, 1982.

---, Purgatorio. Madrid, Visor, 2010.

---, Tu vida rompiéndose, Lumen, Santiago, 2015.

---, Verás, Santiago, Ediciones Biblioteca Nacional, 2016. 
---, “Mentiras verdaderas”, Emisión de La Red, 4 de febrero 2021 Web. Consultado el 25-3-21.

Zurita, Raúl (comp.) y Gander Forest (eds.), Pinholes in the Night: Essential Poems from Latin America, Port Townsend, Copper Canyon Press, 2014.

Zurita, Raúl y Stavans, Ilan (2014) Saber morir: conversaciones. Santiago, Ed. Universidad Diego Portales, 2014.

\section{NOTAS}

1. Ignacio Valente, nombre de pluma del crítico literario, académico y sacerdote José Miguel Ibáñez Langlois, escribe en el artículo mencionado: "En el orden del lenguaje, esta empresa consiste en forjar un idioma poético impersonal, sin sujeto, sin yo lírico, que habla en el código de los teoremas físico-matemáticos, en la forma de los enunciados lógicos más neutros y objetivos (...)". Valente ya había publicado en 1975 "El poeta Zurita" a raíz de la aparición en la revista Manuscritos (1974) de "Áreas verdes", una serie de poemas que será luego incluida en Purgatorio. En esa primerísima reseña hace hincapié en la estructura lógica de los poemas del joven poeta.

2. Si bien existen varios paralelos entre los sucesos sociales y políticos ocurridos en Chile y en Argentina durante las décadas del 70 y el 80, con excepción de una solicitada en reclamo de los desaparecidos publicada en Clarín el 12 de agosto de 1980 a la que Viel Temperley suscribe, la vida y la obra del autor parece mantenerse "al margen" -al menos en el plano de lo explícito- de los hechos políticos nacionales y regionales.

3. Jorge Monteleone resignifica la expresión foucaultiana "la muerte del autor" en los siguientes términos: “¿De qué modo Hospital Británico, no cuenta, para fundamentar su propia singularidad, con la muerte efectiva del autor? ¿No obtiene toda su fuerza de ese hecho documentado, incontestable, que el poema anuncia y el autor cumple?" (2011: 100).

4. En relación al sacrificio de la voluntad, la experiencia de Hospital Británico se diferencia de la de Purgatorio: mientras que el primero conforma una obra en esquirlas, un éxtasis que efectivamente supone un salirse de sí donde regresan distintos versos de obras anteriores y por ende, momentos de una vida que pareciera despedirse, Purgatorio presenta el esfuerzo contrario: parte del disgregamiento, e intenta congregar así los fragmentos de ese yo o sí mismo roto por un golpe que lo fija en la estasis de un presente absoluto. Este tema será ampliado en el anteúltimo apartado del presente artículo.

5. Como ha hecho notar Zurita en su introducción a la obra de Viel Temperley en la antología Pinholes in the Night (2014), la escritura consiste en el intento de expresar el extremo dolor a sabiendas de que el lenguaje no es capaz de expresarlo. Es este intento de formar en palabras aquello que es del orden del grito (el más allá del lenguaje) lo que da cuenta de la voluntad de "participar nuevamente en la vida; esto es, en el purgatorio de las palabras" (Zurita, Gander 2014 Web). Según el autor, es este acto de redención parcial lo que expresa Hospital Británico.

6. Respecto a la relación entre absoluto y absolución, vale aclarar que no se trata únicamente de un vínculo etimológico. Siguiendo a Giorgio Agamben en El lenguaje y la muerte. Un seminario sobre la negatividad, la Voz y la negatividad-conceptos ampliamente analizados a lo largo de ocho jornadas, siete excursus y un epílogo-forman parte, 
precisamente, del entramado conceptual que aúna ontológicamente lo absoluto y la absolución. No nos es posible desarrollar el extenso despliegue conceptual sobre dichas nociones que, según el autor, son centrales, no solo para el estudio de la metafísica occidental, sino también para el análisis de todo lenguaje. En rigor, una de las propuestas del libro es intentar vincular el fundamento negativo del lenguaje con el fundamento negativo de la ontología (66). En esa vinculación, los conceptos antes mencionados juegan un papel central.

7. Al inicio de la primera jornada de El lenguaje y la muerte. Un seminario sobre el lugar de la negatividad, Agamben retoma Ser y tiempo (\$50-53). Allí explica que la comprensión del Dasein como un todo, solo es viable, siguiendo a Heidegger, a partir de la relación con su muerte. Este "todo" se entiende como la reunión de experiencias que pertenecen a una vida cuyo conjunto se define por la clausura que opera la conciencia de su muerte. Escribe Agamben: "Solo bajo el modo, puramente negativo, de este ser-para-lamuerte, en el que tiene la experiencia de la imposibilidad más radical, puede el Dasein tener acceso a la propia dimensión más auténtica y comprenderse como un todo" (2016: 14). Podemos pensar que esta exposición a la propia muerte como la posibilidad de la imposibilidad, es la experiencia que subyace en las obras poéticas acá analizadas en tanto que ambas se escribieron desde, y por lo tanto, habitan, el umbral entre la vida y la muerte. Este hecho las vincula definitivamente con la negatividad, concepto clave para comprender la noción de absolución antes mencionada. La absolución no refiere al ámbito moral sino al ámbito ontológico, es decir, a la asunción de que una o uno morirá. Siguiendo a Heidegger, la culpa -no frente a hechos que impliquen un juicio moral, sino frente al íntimo estremecimiento que surge del vislumbre de la propia finitud- tiene lugar en este silencioso reclamo ( $R u f$ ) de la conciencia y por eso, "es a la vez revelación de una negatividad (Nichtigkeit) que pertenece originariamente al ser del Dasein" (15).

8. En el caso de Zurita, el concepto de trascendencia opera de modo paradojal, es decir, a la manera de "trascendencia sin trascendencia". Para mayor desarrollo de este sintagma, ver "Vínculos entre la poesía de Raúl Zurita y algunas ideas de Jacques Derrida" (Busquet: 2019b). Viel Temperley, en cambio, expone una visión de trascendencia anclada en la fe y en la experiencia concreta de comunión con Dios.

9. La cita completa dice: "No puedo sino trabajar con mis carencias, transformar mis complejos, mis equivocaciones y mis mentiras en obras de arte".

10. Para más desarrollo sobre el movimiento descendente en la obra de Raúl Zurita, ver: "Zurita o el poema exponencial de la piedad" (Busquet 2021).

11. En cuanto al discurso lógico, La nueva novela de Juan Luis Martínez (1977) anticipa algunas de las fórmulas enunciativas de carácter proposicional que se leerán dos años más tarde en Purgatorio, en particular en la sección "Áreas verdes". Como se sabe, ambos poetas compartieron por aquellos años, además de la misma máquina de escribir, una relación de amistad muy cercana.

12. Respecto a la expresión "abajamiento hacia lo alto", en el texto Figura (1938), Auerbach realiza una genealogía conceptual sobre este movimiento descendenteascendente, donde sintetiza la conexión entre lo bajo y lo alto por medio de la expresión sublimitas humilitatis. Para más desarrollo ver: Cuesta Abad en Auerbach 1998: 38. En una dirección similar, Giorgio Agamben retoma este doble movimiento de elevación por medio del descenso en Altísima pobreza. Reglas monásticas y formas de vida (2011). 
13. De manera semejante, en el artículo "Viel Temperley, hombre al agua", Mariana Di Cío señala que el poeta "ha logrado tomar plena conciencia de los límites del lenguaje, y de la imposibilidad de decir el Verbo" (2016: 234).

14. En su libro sobre la experiencia mística en la obra de Viel Temperley, Gabriela Milone también se detiene en la noción de límite: “Ante un lenguaje que designa el mundo, la in-fancia que vuelve al origen. Ante el instrumento lingüístico convencional donde las palabras refieren a las cosas, la experiencia pura y originaria del hombre en sus propios límites. Ante el conocimiento del objeto puesto frente al sujeto que construye representaciones, la autoridad que se vive en y por los límites" (2003: 21). "Así, la poesía como frontera y fronteriza del lenguaje es lo ilimitado de los límites (¿silencio?) o lo limitado de la ilimitación (¿palabra?) hacia donde la experiencia se dirige para su expresión ilimitable (¿intransferible?)" (27).

15. "Esta es la indiferenciación absoluta que puede servir de punto de partida. Indiferenciación donde hay, sin embargo, diferenciación (quiere y no quiere) pero negada" (Fatone 2009: 39)

16. La interpretación cristiana de la figura del los peces también es sostenida por Mariana de Cío en el artículo "Las llagas abiertas de Raúl Zurita" (2019: 53), mientras que Alejandro Tarrab en "Purgatorio el lugar natural de las artes" sugiere que el poema zuritiano está inspirado en el poema "Canción nocturna de los peces" (1905) de Christian Morgenstern.

17. La fórmula "ni ni" se puede rastrear también en el ámbito de la filosofía contemporánea francesa. Maurice Blanchot trabaja este sintagma en el libro El paso (no) más allá (Le pas au-delà) (1973) en vistas de definir el concepto de "lo neutro". A su vez, Jacques Derrida recurre a la lógica del "ni ni" a la hora de pensar algunos de los conceptos principales de su obra como: "el entre", "lo indecidible", "la espectralidad".

18. "Y el que había estado muerto [Lázaro] salió, con las manos y los pies vendados, y el rostro cubierto con un lienzo. Jesús les dijo: -Quítenle las vendas y déjenlo ir" (Juan 11:44).

19. Como indica Max Anselmi: "Para la fe, la función crítica de la cruz consiste en tomar en serio la analogía y la relación que nace y, especialmente, el "vacío" que toda relación y afirmación dejan abierto. El "vacío" (kénosis) no se entiende tanto con relación a las cosas, vistas en su límite, sino en referencia a la misma cruz. En este caso, el "vacío" de la cruz no es otra cosa que el "espacio de libertad", una toma de distancia crítica. Breton escribe: "La fe en la Palabra de la Cruz es el 'vacío de una toma de distancia' respecto a los prestigios del ser, del tener y del poder"' (2004: 73).

20. Jorge Monteleone señala que Hospital Británico: "Es tanto el libro de una agonía y la voz lírica de un muerto, como un poema de resurrección en el Logos cristiano" (2011: 103). Silvio Mattoni también inscribe este libro bajo la figura de la resurrección (2011: 98).

21. En más de una entrevista, Zurita explica que el cierre de su obra -lo que sería en términos cinematográficos "la última escena"- se condensa en los versos de un poema de Anteparaíso (1982: 158), segundo libro publicado por el autor, que aparecen bajo el título "Poema final" en Tu vida rompiéndose (Antología personal) (2015: 590). Estos versos fueron escritos al comienzo de su producción poética, y en ese sentido podemos pensar que nos reenvían a la frase paulina. 
22. Si bien la poética de Zurita cobrará con el tiempo una escala semejante a la obra de Pablo Neruda -especialmente en lo que refiere a la voluntad de totalización y al triple engranaje lingüístico, territorial e histórico- para el año 1979, fecha de publicación de Purgatorio, ese despliegue monumental aún no se había dado. Por último, la organicidad de la obra zuritiana incluye además de los tres planos referidos (lingüístico, territorial e histórico), un cuarto punto: el eje autobiográfico. Este cuarto polo cobra especial relieve en la obra cúlmine Zurita (2011), que según el autor puede leerse como una novela, o podríamos aventurar, como una enciclopedia de sí mismo, un círculo de conocimiento que da plena cuenta de la totalidad de la vida de un hombre sudamericano.

23. Para más desarrollo sobre el límite de la poética nerudiana y la antipoesía de Nicanor Parra, ver: Brockenshire y Busquet, "Zurita: una poética sudamericana del paraíso" (2021).

24. Con un lenguaje teórico y un extenso despliegue conceptual, en el texto Literatura, lenguaje y sociedad: 1973-1983 (1983), Zurita afirma la necesidad de un "nuevo lenguaje" capaz de dar cuenta del quiebre social, político pero también lingüístico que significó el golpe de Estado de 1973. Esta rotura de la columna o espina dorsal del siglo sudamericano -retomando en clave latinoamericana la expresión de Ósip Mandelstam (Badiou, 2005: 33)- significó un punto de irreversibilidad en el régimen de habla y por lo tanto, en sus horizontes poéticos. Siguiendo a Zurita, luego del golpe se pasa del "régimen de conversación" al "régimen de lo no dicho" (1988: 14). Las obras poéticas que de este pasaje se derivan -entre las cuales el poeta ubica sus dos primeros librostienen como eje estructurador del lenguaje, precisamente, la experiencia de la censura que funda "lo no dicho".

25. Escribe William Rowe a próposito de la unión entre paisaje y lógica en el modo de representación de Purgatorio: "El sitio de la no-presencia está ocupado por lo real espacial, de índole lógica y matemática, que nada tiene que ver, insistimos, con el espacio representado, sino que, al contrario, lo destroza" (2017: 17).

26. A su vez, Delfina Muschietti vincula Hospital Británico con "los grandes textos canónicos de los místicos de la tradición cristiana" como San Juan de la Cruz, Santa Teresa, Santa Sor Faustina (2011: 119).

27. "No obstante, unos pocos escritores argentinos han sido considerados, por algunos estudiosos, como "poetas místicos": Jacobo Fijman, María Raquel Adler, Héctor Viel Temperley, Miguel Angel Bustos, Hugo Padeletti, Hugo Mujica, Francisco Luis Bernárdez, Ricardo Molinari, Juan L. Ortiz, Leopoldo Marechal, Alejandra Pizarnik, Olga Orozco y Juan Gelman. Los criterios para tal denominación varían de un crítico al otro" (2020: 17).

28. "La poesía mística, efectivamente, no se ha prodigado en el siglo XX, al menos en nuestro país. Más bien ha predominado la desacralización programática, con la gama completa de los sucesivos descreimientos. (...) En Argentina hay mucha poesía religiosa, pero apenas existe la mística" (Sylvester 2011: 138 y 139).

29. La cita de Novalis se encuentra además en el libro El amor y Occidente (1939) de Denis de Rougemont. El autor vincula la mística y la pasión erótica dando cuenta, a su vez, del desasimiento presente en ambas experiencias: “Un rasgo profundo de la pasión -y de la mística en general- aparece aquí. (...) la pasión no es, en modo alguno, esa vida más rica con la que sueñan los adolescentes; es, muy al contrario, una especie de intensidad desnuda y desposeedora; sí, verdaderamente es una amarga desposesión, un 
empobrecimiento de la conciencia vacía de toda diversidad, una obsesión de la imaginación concentrada en una sola imagen; y a partir de entonces el mundo se desvanece, 'los demás' dejan de estar presentes, no quedan prójimo, deberes, vínculos que se mantengan, tierra ni cielo: estamos solos con todo lo que amamos" (1984: 149).

\title{
RESÚMENES
}

Se ha dicho que Purgatorio y Hospital Británico pertenecen respectivamente a los polos lógico y místico de la poesía latinoamericana de finales del siglo XX. El presente artículo propone establecer que estos dos polos constituyen más bien un continuum, que permite, con diferencias, la subjetivación de una vida por medio de la escritura. En última instancia, estos proyectos poéticos son tentativas de transitar lo intransitable: en Zurita, la experiencia de la dictadura; en Viel Temperley, su enfermedad terminal. Tales circunstancias hacen de estos libros obras catábicas y escatológicas, signadas por una escritura cuyo tránsito reconduce a una suerte de absolución.

On a souvent dit que Purgatorio et Hospital Británico appartiennent respectivement aux pôles logiques et mystiques de la poésie de l'Amérique Latine de la fin du $\mathrm{XX}^{\mathrm{e}}$ siècle. Nous propossons que ces deux pôles constituent en réalité un continuum, permettant -avec certaines différencesla subjectivation d'une vie par l'écriture. En somme, ces deux projets constituent des tentatives de sonder l'insondable: chez Zurita, l'expérience de la dictature, chez Viel Temperley, sa maladie en phase terminale. De telles circonstances font de ces livres des œuvres catabatiques et eschatologiques, marquées par une écriture dont le passage ramène vers une sorte d'absolution.

It has been said that Purgatorio and Hospital Británico pertain respectively to the logical and mystical poles of late-twentieth-century Latin American poetry. The present article aims to establish that these poles form, in actual fact, a continuum that leads, albeit in different ways, to the subjectification of life through writing. Thus, these poetic projects are best understood as attempts to bear the unbearable; in Zurita's case, the experience of dictatorship; in Viel Temperley's, his terminal illness. Such circumstances single these books out as katabatic and eschatological works marked by acts of writing that deliver its authors a degree of absolution.

\section{ÍNDICE}

Mots-clés: poésie de l'Amérique Latine, mystique, eschatologie, poétiques terminales, absolution Palabras claves: poesía latinoamericana, mística, escatalogía, poéticas terminales, absolución Keywords: Latin American Poetry, Mysticism, Eschatology, Terminal Poetics, Absolution

\author{
AUTOR \\ ADELA BUSQUET \\ Universidad de Buenos Aires - CONICET \\ lelibusquet@gmail.com
}

